\title{
Envisioning Internet of Things using Fog Computing
}

\author{
Urooj Yousuf Khan \\ Department of Computer Science \\ SZABIST Dubai Campus \\ Dubai, United Arab Emirates
}

\author{
Tariq Rahim Soomro \\ College of Computer Science \& Information Systems \\ Institute of Business Management (IoBM) \\ Karachi, Pakistan
}

\begin{abstract}
Internet of Things is the future of the Internet. It encircles a wide scope. There are currently billions of devices connected to the Internet and this trend is expecting to grow exponentially. Cisco predicts there are at present 20 billion connected devices. These devices, along with their varied data types, transmission rates and communication protocols connect to the Internet seamlessly. The futuristic implementation of Internet of Things across various scenarios demands the real time performance delivery. These range from RFID connected devices to huge data centers. Until date, there is no single communication protocol available for envisioning IoT. There is still no common, agreed upon architecture. Hence, huge challenges lie ahead. One of the ways to envision Internet of Things is to make use of Fog Networks. Fog is essentially a cloudlet, located nearer to the ground. It offers lower latency and better bandwidth conservation. The Fog or Fog computing is a recent concept. The OpenFog Consortium is a joint effort of many vendors. Its latest work is the background study for realizing Fog as a possible paltform for activating Internet of Things. This paper revolves around Envisioning Internet of Things using Fog computing. It begins with a detailed background study of Internet of Things and Fog Architecture. It covers applications and scenarios where such knowledge is highly applicable. The paper concludes by proposing Fog Computing as a possible platform for Internet of Things.
\end{abstract}

\section{Keywords-IoT; fog computing; cloud computing}

\section{INTRODUCTION}

Internet of Things (IoT) is the future of the Internet. It enables inter connectivity among devices and platforms. It is expected that by 2020, IoT will expand up to 26 billion devices, a huge leap since 2009. One of the main challenges to enabling IoT is the ability to identify each device uniquely. Many techniques exist in this regard. A popular approach is to assign each device, anIPv6 address. This enables the devices to be identified exclusively. On a more advanced level, Uniform Resource Identifiers (URI) can be deployed. These include both IPv6 addresses and Uniform Resource Locators (URLs). URI is used in parallel with Domain Name Service (DNS). Another important domain is that of location sharing and identification. Participating gadgets: Things need to find out one another, approve each other and then share information over a link. Sharing information using protocols and data formats must be common to both participating devices [1]. Uninterrupted Internet connection is ensured by Cloud platforms. The "Pay-As-You-Go" model is an efficient way of managing data centers, for processing client applications and batch processing. This shift in the paradigm towards centralized cloud computing is primarily due to the ease in management. It further includes scalability, expansion of data centers, automatic backup and elevated physical data security. However, uninterrupted Internet connection might be an expensive requirement for many small systems. They could be embedded devices, pervasive systems or simple battery operated circuits [2].

Such devices require mobility, location awareness and low latency. In reality, it might be ideal to have one local device to provide required Internet access to all devices. If the majority of device requests can be entertained locally, IoT vision can be materialized. To meet these requirements, a new platform, namely Fog Computing or simply Fog is coming into perspective. Fog Computing or Fog is a framework where large number of heterogeneous devices collaborate with each other to perform various networking tasks within the network, without the intervention of third party. The purpose of the study is to explore the potential implementation of IoT. It discusses Fog as a possible platform for enabling IoT [3].

Fog is essentially a cloudlet, placed near to host device [2]. It enables devices to identify and interact with each other locally. It thus prevents the redundant cloud access. This, in turn, leads to efficient data processing and better security. Fog is still a newer concept and is in its primary development phase. The paper is organized as follows: Section 2 covers the Literature Review; Section 3 discusses Architecture for Internet of Things, while Section 4 discusses Cloud Computing and the Fog. Findings are covered in Section 5. Discussion and Future work is covered in Section 6.

\section{LITRATURE REVIEW}

The term Internet of Things was first coined by Kevin Ashton in 1999 [4]. It is an effortless interweaving of sensors, actuators and drivers. It is a concept that has taken firm grounding in the recent research years. IoT incorporates universal communication using existing, valid protocols. It does so by uniquely addressing each of the participating devices by using smart, interactive interfaces. Future Web i.e. the next generation of the Internet combines many aspects such as Internet of Things (IoT), Intelligent Networks (IN), Internet of Service (IoS), Internet of Content (IoC) and Internet of Media (IoM) [5]. Many technologies support this concept. These include Radio Frequency Infrared Detector (RFID), Near Field Communication (NFC), Optical tagging and Quick Response (QR) codes and assigning an IPv6 addresses to each device. As the web progresses from static, HTML based websites to more dynamic, AJAX-enabled (Asynchronous JavaScript and XML) Web sites and social networking towards Ubiquitous Computing, the need for smart 
representation and all-time availability of data has increased by many folds [4].

RFID was one of the oldest in this regard. To be exact, a new Ultra High Frequency (UHF) RFID tag standard was created by EPC (Electronic Product Code) Global. The aim was to replace bar codes with machine-readable ones that could be read from a distance. The idea, however could not take firm ground due to limitations such as poor product design. Another visualization for Internet of Things came from Near Field Communication (NFC). Although currently limited, the scope for this field is bright. It is a step forward in RFID. The purpose for this implementation was to enable smart phones to read passive NFC tags. These tags could store Universal Resource Identifier (URI).They were portable, thin and small chips that could be attached to any device. Apple's share in this regard was also significant. In September 2014, Apple announced that iPhone6 would embed NFC support, promoting NFC to be a key player in IoT. A varied implementation for IoT comes from Optical tagging or Quick Response (QR) tags. These tags can serve as identifiers for devices. QR is particularly popular as every smart phone is equipped with a high-resolution camera. A QR code is extracted and read from the scene using the image-processing techniques. These methods yield a hidden text, number or URI. These QR codes are found in numerous products [1].

None of these technologies, however, has the scalability to fully enable Internet of Things. The scope and application of IoT are enormous. The major areas include agriculture, aerospace industries, environmental technology, and intelligent, embedded systems to name a few [5]. The participating devices range from very small Wi-Fi enabled devices to huge data centers. The key cost effective factors include size and battery requirements of the embedded devices. In order to reduce battery consumption and increase device portability, chip designers aim at producing smaller and more independent devices at a minimum investment [2].

The term "Cloud Computing" became popular after Google's CEO Eric Schmidt used it. It is another paradigm in the field of research. According to National Institute of Standard and Technologies (NIST), Cloud Computing is defined as "a model for enabling ubiquitous, convenient, ondemand network access to a shared pool of configurable computing resources that can be rapidly provisioned and released with minimal management effort or service provider interaction". Cloud services have gained popularity over last few years. It is due to the fact that Cloud services provide ease of management and lesser hassle on the client's end. It is a platform in which system assets namely processor, memory, bus and bandwidth are provided as utilities that can be claimed and released per the user need via Internet. This low cost model with seemingly infinite storage and processing power has gained huge market acceptance over very short period. It is evident from the fact the tech-giants like Google and Amazon have shifted their services to Cloud. Cloud computing contains certain relatable features such as resource provisioning. A layered model of Cloud computing reveals 4 layers. These are the hardware layer, the infrastructure layer, the platform layer and the application layer [6], [7]. Cloud services fall into three layers of the stack: Infrastructure as a service (IaaS), platform as a service (PaaS) and software as a service (SaaS). IaaS is the most basic category. It permits the purchase of IT infrastructure from a cloud-provider. PaaS implies the deployment of cloud computing services for software development. It enhances development, testing and managing software. The developers need not worry about underlying details of servers, networks and databases. SaaS is a scenario where delivered over the internet on subscription basis [8].

It is imperative here to understand the various Cloud deployment scenarios as well. They also fall into three major classes: Public, Private and Hybrid. Hybrid clouds combine public and private clouds, permitting greater flexibility and data sharing. In spite of the fact that Cloud services provide reduced cost and better development, there are certain downsides of it. Latency and intermittent connectivity are major contributors. Poor bandwidth results in slower performance and affected quality [9]. Security and nonnegotiable terms of services also become bottleneck in production environment. Lack of support, minimal flexibility and limited knowledge about the system hardware can also become a retarding factor in visualizing Cloud services to its full potential. In certain applications such as health monitoring and emergency response, delay and downtime cannot be tolerated. Fog answers these downfalls using nearer to ground connection and larger coverage area. Fog computing brings the Cloud closer to the ground. It is a paradigm of managing a largely available, varied data requirements and data sets [10].

The purpose of Fog computing or fogging is to enhance efficiency and lessen the amount of data travelling to the cloud. It does so by entertaining the majority of device requests itself without forwarding them to the cloud. It results in better security and efficient utilization of bandwidth. In a Fog scenario, data processing takes place in a data hub or local gateway router. It is worth mentioning here that Fog Network complements the Cloud computing. It provides quick, shortterm response and analysis at the edge. It provides deeper insight to system as multiple data points provide it with data [11]. Fog computing can be an ideal platform for enabling Internet of Things as it is the cloud nearer to ground [3]. Fog devices can be termed as nano Data Centers (nDCs). These are tiny servers located at deployment site. These nDCs can distribute data at the client site and entertain client requests locally [12]. If Internet of Things is implemented using Fog computing, the vision of IoT can be realized. It will enhance larger number of applications and practical scenarios [6]. In this study, we discuss a possibility of implementing IoT using Fog. It proposes a possible platform for IoT using Fog computing.

\section{ARCHITECTURE OF INTERNET OF THINGS}

The research approach adopted in this paper is qualitative nature backed by literature review and analysis. Based on this pattern we derive a nearly predictable, temporal conclusion of implementing IoT using Fog. Majority of the papers reviewed are published during 2008-2017 [6]. 


\section{A. Internet of Things Framework}

IoT is still in its infancy. It is based on a multi-layered architecture. This layered approach divides the functionality such that varied requirements of different industries and businesses can be met [5]. The precise and accurate layered structure of IoT is still debatable and there is no common agreed-upon model for its implementation. Largely, IoT comprises of at least three layers: Perception, Network and Application Layer [13]. This model was developed in the early stages of its development. It defines the main theme of IoT but does not cover its implementation details very precisely.

- The Perception Layer: It is the physical layer. It comprises of sensors and actuators in the physical environment. The purpose of this layer is to gather the data from the environment. The main task of this layer is to gather data from various sources. These sources are heterogeneous in nature, ranging from 6LoWPAN to embedded systems deploying RFID or Optical tags.

- The Network Layer: This layer is all about connectivity. It works as a linking layer among various devices. It serves to connect smart devices, network hubs and servers. It is also responsible for transmitting and processing data received from various layers.

- The Application Layer: This layer is responsible for entertaining the user requests. It defines various scenarios in which IoT can be deployed.

These layers are further elaborated in the five-layer model as depicted in Fig. 1.

A more detailed layered approach is a five-layer model. It additionally includes two more layers of processing and business. The remaining three layers are discussed as follows:

- Transport Layer: It moves data from lower perception layer to higher layer for processing. It does so by deploying various network techniques such as Wireless, 3G, LAN, Bluetooth, RFID, etc.

- The Processing Layer: This layer is also known as the middleware layer. Its task is to store, analyze and process huge amount of data that comes from the lower layer i.e. the transport layer.

- The Business Layer: It manages the whole IoT system, including applications, deployed business models, etc.

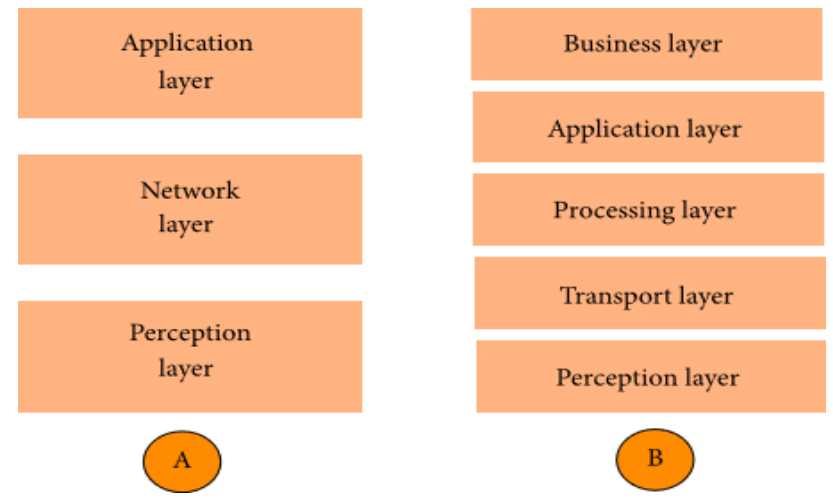

Fig. 1. Layered architecture of IoT [13].

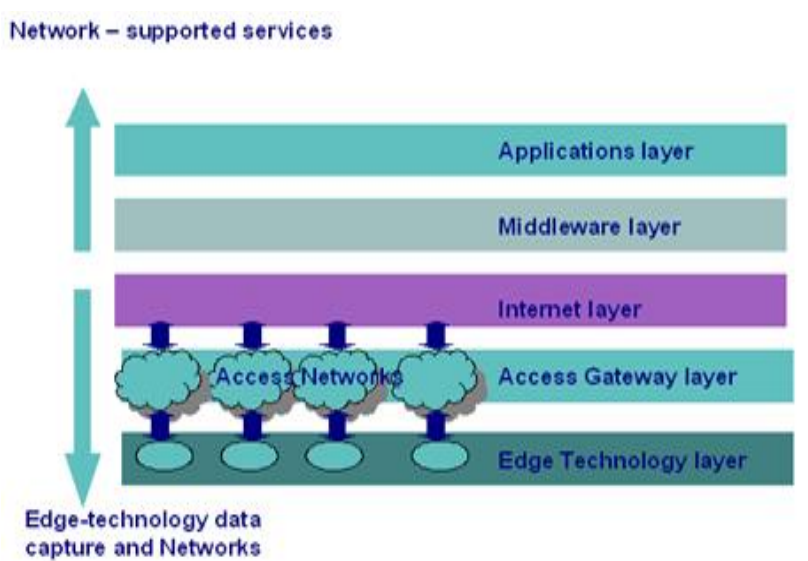

Fig. 2. Alternate architecture for IoT [5].

Another way to describe the layered architecture of IoT is by using Top-down approach. The layers essentially have the same functionality. This particular architecture focuses on standardization and interoperability [14]. This model has following layers: Application Service layer, Utility layer, IoT Service layer, and the Environment layer. Another literature describes this architecture as Technology Edge layer, Access Gateway layer, Middleware layer, Application layer and Internet layer [5]. These layers essentially perform the same functions.

Technology Edge layer is the physical layer comprising of sensors and actuators and are depicted in Fig. 2.

- Access Gateway layer is the network layer.

- Middleware layer is transport and processing layer together.

- Internet layer and Application layer are domain specific and application dependent.

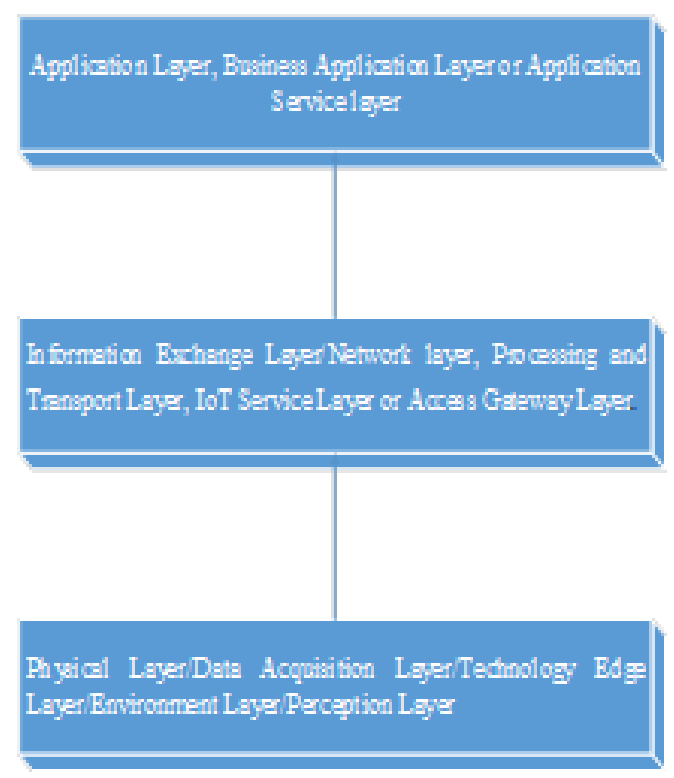

Fig. 3. Consolidated layered approach for IoT. 
Another taxonomy describing the layered architecture of IoT breaks down the layers into Data Acquisition Layer, Information Exchange Layer and Application Layer [15] and as shown in Fig. 3. Let's explore the functionality of each layer in detail.

\section{B. Data Acquisition Layer}

This layer is referred as the Physical Layer, Technology Edge Layer, Environment Layer or the Perception Layer. This is the layer closest to input devices. It is the layer that captures the user data. It comprises of multiple identification schemes and methods. Input devices include Bar Code Readers, RFID tags, Global Positioning System (GPS), sensors and actuators, embedded devices, Wireless Sensor Networks (WSN), Low-powered Wireless Personal Area Network (6LoWPAN) or simple Wi-Fi enabled devices. The main purpose of this layer is to capture data from the user in real time. It also must have object identification system. It comprises of multiple data acquisition methods and modules. It serves to complete the information perception of the IoT. It does so to provide a solid ground for the above layers in the model. Here the main challenge is to manage device heterogeneity and ability to capture large amount of data in real time.

\section{Information Exchange Layer}

This layer is termed as Network layer, Processing and Transport Layer, IoT Service Layer or Access Gateway Layer. This layer is the heart of enabling IoT. It comprises of various communication technologies that form the backbone for envisioning IoT. Defining technologies here include network routing protocols, mobile communication technologies and the Internet. It is at this layer that rich information exchange and routing takes place. It includes information processing, information management and data convergence. This layer handles huge, intelligent and varied data. It is at this infrastructure layer that the vision of IoT can become a universal reality. A key player in this layer of information processing and management is Cloud. Cloud computing has already revolutionized the way information is stored, processed and delivered to business clients.

\section{Application Layer}

This layer is termed as Application Layer, Business Application Layer or Application Service layer. It refers to the merging of various IoT solutions and technologies with industries. This layer addresses wide range of industrial problems. It is through this layer that IoT realizes its deep connection with real world. It includes a variety of servers. Its main function is the analysis of gathered data, adaptation to user needs, socialization and security of the data [15].

\section{Cloud COMPuting AND THE Fog}

The term Cloud computing has taken the industry by storm. It essentially means storage and access of data and instructions over the Internet [16]. It is a general term used for services that are controlled, managed and delivered through the Internet. It equips the companies with resource sharing and virtually infinite memory. Companies pay to the services provider by assuming Internet as a utility. It frees organizations to focus on Solution design rather than worrying about maintaining the computing infrastructure [17].

\section{A. Cloud Computing Architecture}

This section discusses in detail the underlying architecture and various deployment models for Cloud. The model for Cloud computing follows a modular or layered approach. The top and the bottom layer lightly bind each of the participating. It simplifies understanding and provides service-based understanding of the Cloud. The layered architecture also enhances modularity of resources. It means that the owner of each layer has its own milestones. Broadly speaking, the Cloud is divided into four layers: The Hardware/Datacenter Layer, the Infrastructure Layer, the Platform Layer and the Application Layer [18], [19]. These layers are depicted in the Fig. 4.

Here we describe each of these in detail:

- The Hardware Layer: This layer is implemented at the Data Centre and is responsible for all the physical devices, nodes or computers connected to the Cloud. These resources include servers, routers, switches, power and cooling systems, etc.

- The Infrastructure Layer: This layer is also termed as Virtualization Layer. It is a core layer in Cloud as it provides dynamic resource management. It creates a huge, virtual pool for hardware resources.

- The Platform Layer: Next in the stack is the Platform Layer. It consists of Operating Systems and Application frameworks. Its main purpose is to reduce the workload of the Infrastructure layer.

- The Application Layer: This layer is the top most layers. It contains the actual Cloud applications. These are different from traditional ones as they provide scalability and Bandwidth conservation.

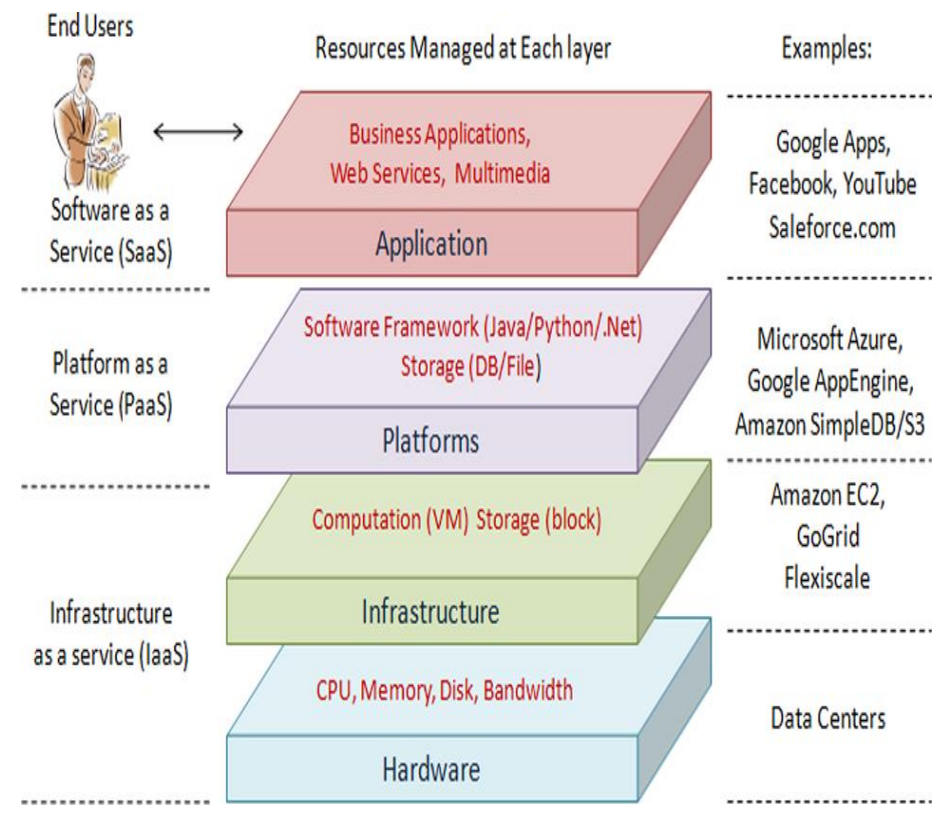

Fig. 4. Layers in a cloud [18]. 


\section{B. Disadvantages of Cloud Computing}

Following are the main disadvantages of Cloud Computing [20] :

- Downtime: It is in fact the biggest downside for Cloud computing. Since Cloud services are dependent on the internet connection, it implies a loss of Internet connection can bring the entire service down. One of the most notable incidents took place in 2014, where DropBox faced an outage of around two days.

- Privacy and Security: By outsourcing the company data to an external provider, the company faces security and risk issues. One of the most notable examples was Code Space which was forced to close down after its console was hacked.

- Vulnerability to Attack: Nothing connected to the internet is perfectly secure. Even the best of teams and the most secure of the systems face cybercrimes and Vulnerability attacks.

- Limited Control and Flexibility: Cloud users have coarse grained control over the services provided by the Vendor. They often lack flexibility and agility.

- Platform Dependency: It is also termed as "Vendor Lock-in". Finer differences between different vendor systems sometimes make migration to a newer platform very difficult.

- Cloud Computing Costs: For smaller scales and short term projects, Cloud can be hefty.

With the above mentioned disadvantages, it can be seen that there are certain downsides of the Cloud that need to be improved. But even with those downsides, Cloud has huge potential and scope for enhancing businesses and enterprise.

\section{The Fog}

Fog computing is a term originally coined by Cisco [21]. Fog network are defined by their nearness to the Things and "Computational Density at the Edge of the Network". An alternate definition is "system-level horizontal architecture that distributes resources and services anywhere along the continuum from Cloud to Things [22]." In contrast to Edge Computing, a fog may perform analysis on anything in the network from the core to the edge. Fog is essentially an extension of the Cloud, nearer to ground. The connected devices are called Fog nodes that can be placed anywhere in a Control System. Ideally, any smart device i.e. device with computational power, memory and network connectivity can be a Fog device. These typically include switches, routers, embedded sensors and surveillance cameras [23]. The applications can be developed utilizing specific, tiny pieces of code with minimum or no interaction to the Cloud [2]. It has following distinguishing features [22]:

- Horizontal Architecture: It distributes the services and applications horizontally along the industries and enterprises.

- Cloud-to-Thing Continuum: Real-time response for the Connected Vehicles and Smart grids. Bringing
Internet closer to Things. It is in fact, the connection between the Things: sensors, actuators, and Internet in real-time.

- Location Awareness: The participating Nodes are location sensitive and fully aware of their surroundings.

- Low Latency: Fog provides lower latency as it entertains the devices requests locally and in Real-time.

- Wireless Access: The participating devices are equipped with wireless sensors and receivers.

\section{How does Fog Work?}

It follows a layered structure. It is divided into three layers: User Device Layer, Edge Node or the Fog Layer and finally the Cloud Layer [24]. They are shown in Fig. 5 and described below:

- User-Device Layer: It is the layer closest to the Things or the participating nodes.Here the data generated by the node is collected. It can be called as the Physical layer as well. It deals with various types of sensors,grids and real-time processing applications.

- Edge-Node Layer or the Fog Layer: It is the heart of the entire architecture. Here the data is stored, interpreted and analyzed. The most latency sensitive aplications are processed immedialtely. These may include grid sensors or connected vehicles input. Here the data can be checked for errors and redundancy. Data that can tolarate delay is sent for analysis and action to aggregation node. Data collected for long term stydying and prediction is sent to Cloud. It is also possible that plenty of Fog nodes collect similar data and send periodic updates for analysis and design study.

- The Cloud Layer: This layer is essentially the Cloud,described in the previous section.

A more detailed approach to Fog is shown in Fig. 6 and described below:

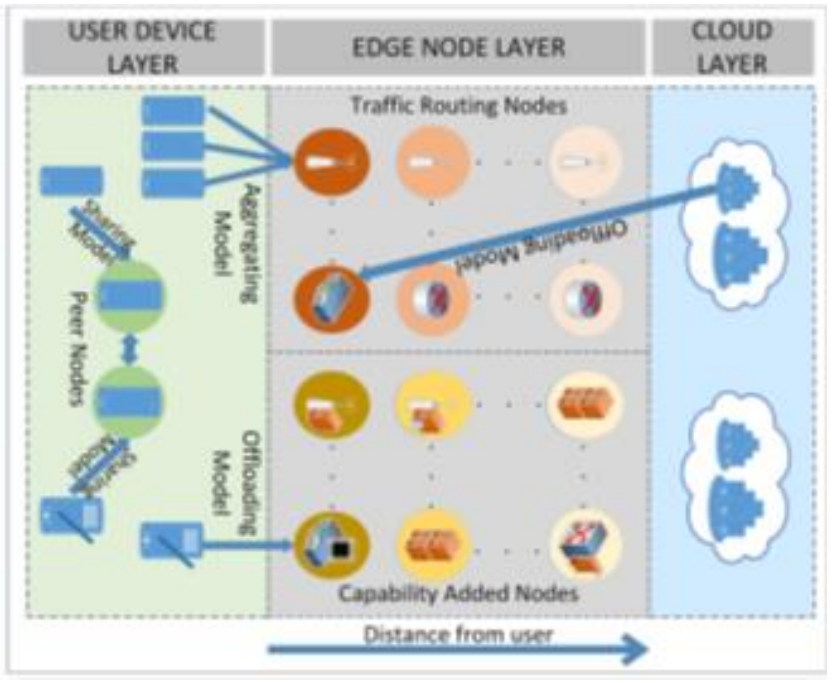

Fig. 5. Fog devices and the cloud [24]. 


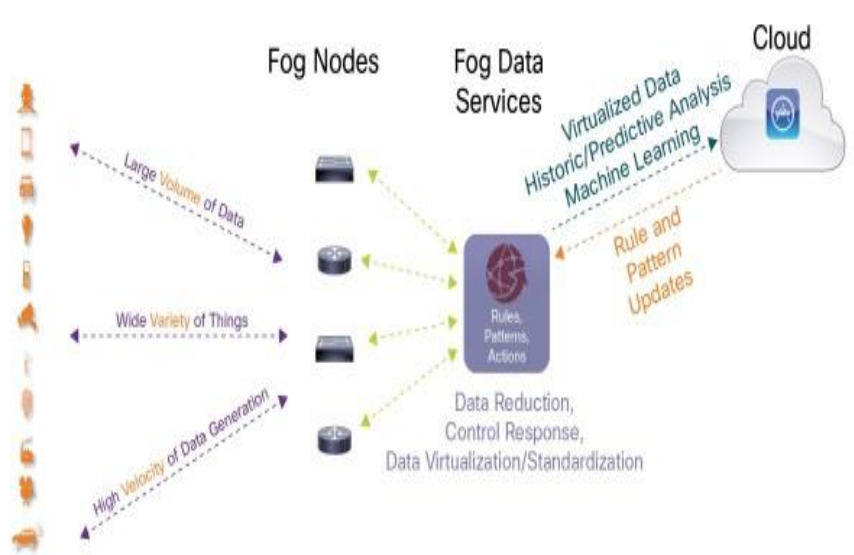

Fig. 6. Fog architecture.

TABLE I. COMPARATIVE ANALYSIS BETWEEN Cloud AND FoG

\begin{tabular}{|l|l|l|}
\hline Feature & Cloud & Fog \\
\hline Downtime & Greater Downtime & Lowest possiblity \\
\hline Connectivity & Fewer devices & $\begin{array}{l}\text { Multiple heterogenous } \\
\text { devices }\end{array}$ \\
\hline Platform Dependibility & Platform dependence & Platform independence \\
\hline Flexibility and Control & Coarse-grained & Fine-grained \\
\hline
\end{tabular}

The layers are essentially the same.

- The first layer or first tier,closer to the node is designed for Human To Machine (H2M) interaction. It enables the data collection,processing and control of the data.

- The second and third layer or tier is designed for Machine to Machine (M2M) interaction.It essentially performs data analysis,error detection [3].

Table I sums up the advantages of Fog over Cloud.

\section{RESULTS AND FINDINGS}

As discussed above, the various Things, or devices that are connected to the IoT are heterogeneous in nature. They range in size, data type and communication speed. Most of them embody real-life applications and connecting them directly to the Cloud would be practically impossible. To handle the volume, variety and velocity of the IoT devices, we need a novel computational and analytical edge namely Fog. Traditional Cloud architecture does not meet all of these requirements completely. Moving the complete data to Cloud adds latency, consumes bandwidth and can seriously cripple Real-time applications. Moreover, Cloud communicates only based on IP and not on any other protocol used in industries. Thus, the realistic approach signifies that the IoT data must be processed and analyzed near the nodes. We call this scenario Fog Computing or simply the Fog. There are many valid reasons for it as already described.

\section{A. Conceptual Model of IoT using Fog Computing}

Devices can be equipped with IoT-based applications, specifically for Fog nodes as shown in Fig. 7 and 8. The devices closest to these can handle this data and based on the required response, decide the ideal location. The time-critical applications are processed first, nearest to the nodes, and then the data that can bear delayed processing or requires multiple inputs or multiple samples of input stream is sent to the level above. Data that requires analysis and long-time storage is sent to the Cloud. This layered approach reduces the latency time and speeds up the processing of the information. Response time can be reduced from minutes to seconds, days and weeks can be mapped in the similar analogy. The time for which the data is stored on the Cloud can also be adjusted accordingly.

Once at the Cloud, the received data can be aggregated, analyzed and summarized; from many Fog nodes to gain better insight into business applications, Big Data analysis and predicting the expected device/system response. These details can be further used to control the device modelling behavior and system response in general. There are great benefits associated with this approach. It promotes better business agility, enhanced security, much finer privacy controls and security and a lower operating expense.

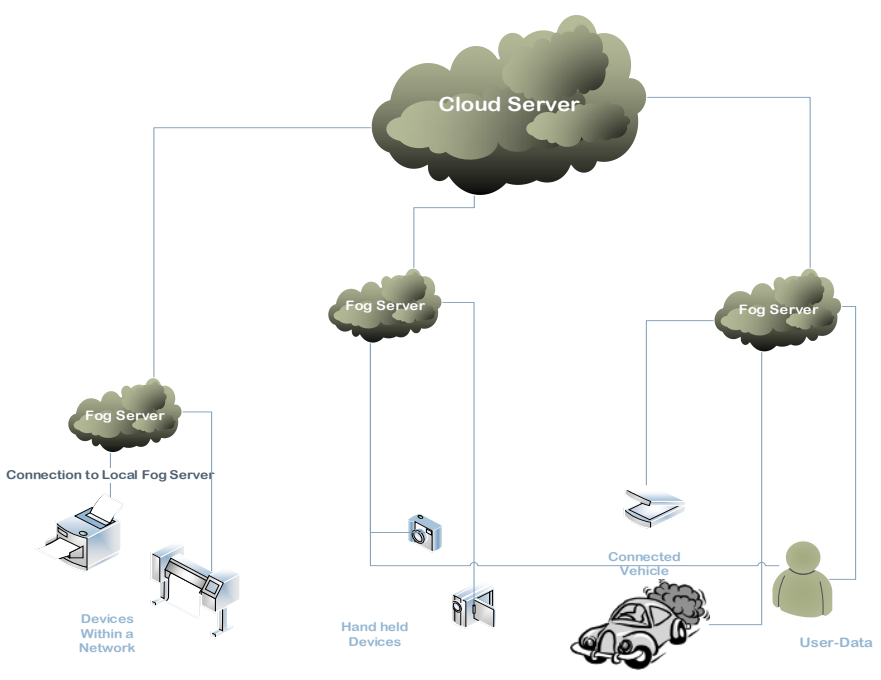

Fig. 7. Conceptual model of IoT using fog computing.

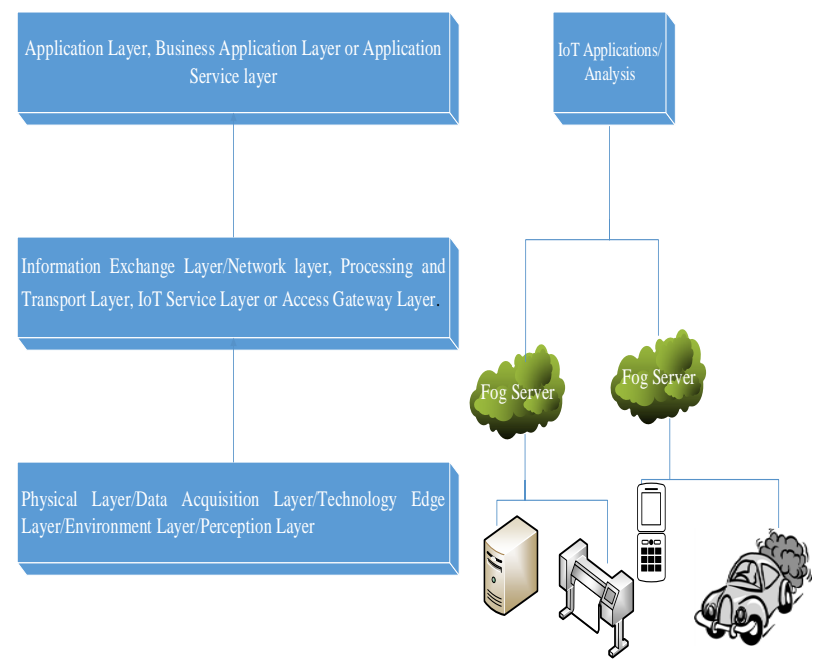

Fig. 8. Mapping IoT layers to devices. 


\section{B. Internet of Things Architecture and the Fog}

The proposed architecture for the Fog computing in IoT is depicted in the Fig. 9 below:

The Internet of Thing Architecture and Fog Computing

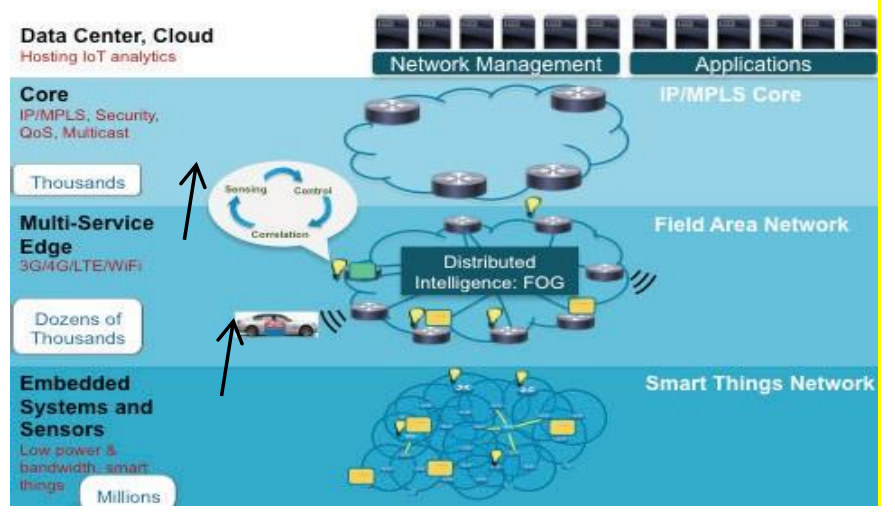

Fig. 9. The Internet of Things architecture and fog computing [3].

Beginning with the bottom most layer, the first layer is of "Things". This layer defines the Human-to-Machine interaction. The first layer of this architecture collects the data and sends control instructions to the actuators. Here is the rich interconnected network of internet-connected devices. The second layer deals with Machine-to-Machine interaction, system processing, reporting and visualization. Here the time scale ranges from microseconds to minutes. This is the core Fog layer. Consequently, Fog must support a number of storage types and multiple inputs at the same time. It must also have wide geographical coverage and broader time scale. The Cloud provides the top most level coverage and data transmission. It is also used as a central repository for data storage; the storage capacity of which is virtually infinite and unbounded by time [3].

\section{Benefits of Fog Computing}

There are numerous benefits of Fog. Some of the advantages are discussed below [23].

- Greater Flexibility for business development. Fog applications can be developed quickly and are more tailored for user needs.

- Better Security: Since majority of the data remains localized, the chances of identity theft and misuse are reduced.

- Finer Control: A finer control can be implemented to access, analysis and control of data.

- Reduced Operational Cost: Network Bandwidth can be conserved, as there is less data transfer to the Cloud.

\section{DISCUSSION AND FUTURE WORK}

The implementation of IoT using Fog computing is a still a new field and is in its infancy. Huge challenges lie ahead. These include definite layered architecture for IoT, communication technologies, Data and Signal processing technologies, Hardware modifications, Network technologies to name a few [5], [22].
- Definite Layered Architecture: The definite architecture and layered approach for IoT is still debatable. There is no set or standard that clearly defines the IoT layers.

- Communication Technologies: Since the participating devices are heterogeneous in nature, there is a lack of common communication technologies and protocols.

- Network Technologies: Lack of networking topologies, technologies and infrastructure in general is a major field of study, still unexplored.

Besides, Fog and its layered structure is still debatable. Hence, implementing IoT using Fog is a major field of research for future. If implemented successfully, IoT can revolutionize the way Internet operates today. Few application areas where IoT can be significant are discussed below:

\section{A. Transportation}

In 2016, it was predicted that an average person produces $650 \mathrm{MB}$ of data every day and it is expected to double soon in future. IoT is important in transportation as it enables the creation of connected vehicle. It enhances low latency, user privacy and resource sharing at different layers. Information collection during peak hours, downloading content while travelling by pooling resources, tracking the position of the vehicle, surveillance cameras on roads and ticketing system in public transport can be visualized. These sub-applications can be entertained locally using Fog computing instead of sending updates to the Cloud. Similarly, safety systems can be activated. The tiebreaker here, the Fog preserves Bandwidth and provides response in Real-Time. Cloud can provide analysis and results such as which routes to choose. A Fog computing scenario for Connected Vehicle is described here [25]. Smart, self-directed vehicles will generate huge amount of data from various sources such as Light Detection and Ranging (LIDAR), Global Positioning System (GPS), etc. Here, the Things cover a broad spectrum: various types of sensors such as roadside sensors, on-vehicle sensors, numerous systems, associated data and functions. These Fog devices also manage actuators. In-vehicle Fog nodes can provide other certain services such as infotainment, advanced driver assistance systems (ADAS), possible collision detection and avoidance. The participating technologies include Dedicated Short Range Communications (DSRC), 3G, LTE etc. These technologies ensure connectivity and network availability [25].

\section{B. Smart Cities}

The infrastructure and design of new cities can be greatly enhanced if IoT is visualized. Envisioning it using IoT can enhance basic city operations such as security, broadband connectivity and safety. Most of the cities provide internet access that leaves little room for the high-ended maintenance services, disaster management advanced municipal services [22]. Smart city is a mega application of IoT as it includes multi-dimensional data and various processing speeds. This majorly includes camera surveillance and camera deployments in places and remote areas that do not have Internet connectivity or Network coverage. This type of coverage is needed for uploading the collected videos, Real-time 
monitoring and anomaly detection. Security and anomaly detection pose a significant challenge in this application. Intrusion detection, elderly health care and fire alarm are all low latency applications that require timeliness and quick response. Moreover, image identification is another addressable context. It implies that while capturing image data, confidential contextual data should not be made public. Fog enables such scenarios to be envisioned accurately and efficiently due to its close proximity with the participating nodes and horizontal distribution. Vector Algorithms, Video analysis and concurrent application processing can hasten the current applications. This is a brief application of Fog in Smart cities. The concept is further applied as smart parking detectors, shopping infrastructure, interlinking hospitals for greater and better services, intelligent highways, and factories that are all interconnected [25].

\section{Smart Buildings}

Building automation and control are also model cases that demonstrate utilization of IoT. There may be thousands of sensors for recording various parameters such as temperature, humidity and parking space [22]. Smart buildings contain a rich interwoven system of sensors and actuators. They can be used to store different parameters such as temperature, humidity, number of people currently in the building, fire detectors, security and alarm. Some of the data is latencysensitive and cannot be delayed. This data is to be analyzed and calculated locally. Typical examples include Security Breach and Fire Alarm [25].

\section{REFERENCES}

[1] B. N. S. a. S. J. Roy Want, "Enabling the Internet of Things," THE IEEE COMPUTER SOCIETY, 2015.

[2] L. R.-M. Luis M. Vaquero, "Finding your Way in the Fog: Towards a Comprehensive Definition of Fog Computing," ACM SIGCOMM Computer Communication Review, October 2014.

[3] R. M. J. Z. S. A. Flavio Bonomi, "Fog Computing and Its Role in the Internet of Things," CISCO, Helsinki, Finland, August 17, 2012.

[4] R. B. S. M. M. P. Jayavardhana Gubbia, "Internet of Things (IoT): A vision, architectural elements, and future directions," Future Generation Computer Systems, vol. Volume 29, no. Issue 7, p. Pages 1645-1660, September 2013, .

[5] M. U. R. S. Gul Ahmad, "INTERNET OF THINGS (IOT): AN OVERVIEW," Journal of Information \& Communication Technology, Vols. Vol. 10, No. 1, no. Vol. 10, No. 1, pp. 122-130, Spring 2016.

[6] W. d. D. ,. V. P. ,. A. P. Alessio Botta, "Integration of Cloud computing and Internet of Things: A survey," Future Generation Computer Systems, p. 684-700, 3 October 2015.

[7] L. C. ,. R. B. Qi Zhang, "Cloud computing: state-of-the-art and research challenges," J Internet Serv Appl (2010), vol. 1, pp. 7-18, 2010.

[8] "https://azure.microsoft.com/en-in/overview/what-is-cloud-computing/," MICROSOFT , Saturday June 2017. [Online]. Available: https://azure.microsoft.com/en-in/overview/what-is-cloud-computing/. [Accessed 10 June 2017].

[9] U. Singh, "https://www.linkedin.com/pulse/11-pros-cons-cloudcomputing-everyone-should-know-umesh-singh," LinkedIn, April 27, 2015 April 27, 2015 April 27, 2015. [Online]. Available: https://www.linkedin.com/pulse/11-pros-cons-cloud-computingeveryone-should-know-umesh-singh. [Accessed 10 June 2017].
[10] H. G. R. N. C. S. K. G. R. B. Amir Vahid Dastjerdi, "Fog Computing: Principles, Architectures, and Applications," in Internet of Things: Principles and Paradigms, ELSEVIER, 28 Jan 2016, pp. 61-75.

[11] M. Rouse, "http://internetofthingsagenda.techtarget.com/definition/fogcomputing-fogging," TECHTARGET NETWORK, December 2016 December 2016. [Online]. [Accessed 10 June 2017].

[12] K. H. ,. R. A., , T. A. ,. a. R. S. T. Fatemeh Jalali, "Fog Computing May Help to Save Energy in Cloud Computing," IEEE Journal on Selected Areas in Communications, 2015.

[13] P. S. a. S. R. Sarangi, "Internet of Things: Architectures, Protocols, and Applications," Journal of Electrical and Computer Engineering, Vols. Volume 2017, , p. 25, 26 January 2017.

[14] M. S. S. V. Bhagyashri Katole, "Principle Elements and Framework of Internet of Things," International Journal Of Engineering And Science, Vols. Vol.3, Issue 5, no. Vol.3, Issue 5, pp. 24-29, July 2013.

[15] B. L. P. D. Hong ZHOU, "The Technology System Framework of the Internet of Things and its Application Research in Agriculture," Funding Project for Academic Human Resources Development , Beijing, 20102011.

[16] B. P. M. M. TEAM, "http://me.pcmag.com/networkingcommunications-software-products/1758/feature/what-is-cloudcomputing," Altus Inc, APRIL 18, 2015, 3:20 P.M. APRIL 18, 2015, 3:20 P.M. APRIL 18, 2015, 3:20 P.M.. [Online]. Available: http://me.pcmag.com/networking-communications-softwareproducts/1758/feature/what-is-cloud-computing. [Accessed 11 June 2017].

[17] S. J. B. $\quad$ Margaret "http://searchcloudcomputing.techtarget.com/definition/cloudcomputing," TECHTARGET NETWORK, October 2016 October 2016. [Online]. Available: http://searchcloudcomputing.techtarget.com/definition/cloud-computing. [Accessed 11 June 2017].

[18] Q. Z. · L. C. . R. Boutaba, "Cloud computing: state-of-the-art and research challenges," Springer:J Internet Serv Appl (2010), vol. 1, pp. 7-18, 2010.

[19] K. M. Yashpalsinh Jadeja, "Cloud Computing - Concepts, Architecture and Challenges," in 2012 International Conference on Computing, Electronics and Electrical Technologies [ICCEET], Gujarat, India, 2012.

[20] S. Seshachala, "https://cloudacademy.com/blog/disadvantages-of-cloudcomputing/," Cloud Academy Blog, 17 Mar, 201517 Mar, 20152015. [Online].

Available: https://cloudacademy.com/blog/?s=Disadvantages+of+Cloud+Computin g. [Accessed Tuesday,13 June 2017].

[21] J. McKendrick, "Fog Computing: a New IoT Architecture?," RTInsights, 2017. [Online]. Available: https://www.rtinsights.com/whatis-fog-computing-open-consortium/. [Accessed 13 June 2017].

[22] OpenFog, "https://www.openfogconsortium.org/resources/," OpenFog, [Online]. https://www.openfogconsortium.org/resources/\#definition-of-fogcomputing. [Accessed 14 June 2017].

[23] "www.cisco.com/c/dam/en_us/solutions/trends/iot/docs/computingoverview.pdf," CISCO, 2015. [Online]. Available: www.cisco.com/c/dam/en_us/solutions/trends/iot/docs/computingoverview.pdf. [Accessed 13 June 2017].

[24] N. W. D. S. N. Blesson Varghese, "Feasibility of Fog Computing," 19 Jan $2017 \quad 19$ Jan $2017 \quad 19$ Jan 2017. [Online]. Available: https://www.openfogconsortium.org/wp-content/uploads/VargheseFogComputing.pdf. [Accessed 13 June 2017].

[25] O. Consortium, "https://www.openfogconsortium.org/wpcontent/uploads/OpenFog_Reference_Architecture_2_09_17-FINAL1.pdf," OpenFog Consortium, February 2017. [Online]. Available: https://www.openfogconsortium.org/ra/technical-document-download/. [Accessed 19 July 2017]. 